\title{
Polysaccharides and mucin 5AC (MUC5AC) expression in gallbladder mucosa of young patients with gallstones as evaluated by spatial visualization and quantification
}

\author{
Aldona Kasprzak ${ }^{1}$, Wojciech Malkowski ${ }^{1}$, Celina Helak-Łapaj ${ }^{2}$, \\ Agnieszka Seraszek $^{2}$, Elżbieta Kaczmarek ${ }^{2}$, Agnieszka Adamek ${ }^{3}$, Maciej Zabel ${ }^{1}$
}

\author{
${ }^{1}$ Department of Histology and Embryology, University of Medical Sciences, Poznań, Poland \\ ${ }^{2}$ Department of Pathomorphology, University of Medical Sciences, Poznań, Poland \\ ${ }^{3}$ Department of Infectious Diseases, University of Medical Sciences, Poznań, Poland
}

\begin{abstract}
The study aimed at examination of tissue expression of polysaccharides and secretory mucin 5AC (MUC5AC) in young patients (up to 25 years of age) with a symptomatic gallstones. For comparison, patients most frequently subjected to cholecystectomy were studied, i.e. patients of approximately 50 years of age with the same diagnosis. In quantitative studies on tissue expression of both mucus components, the modern technique of spatial visualization was applied for the first time. Application of the technique permitted to demonstrate significant positive relationships between expression of glycoproteins (immunocytochemical $\mathrm{ABC}$ technique for detection of MUC5AC) and expression of sugar components in mucus (PAS technique) and to confirm suitability of the technique for quantitative appraisal of both histochemical and immunocytochemical reactions. An even higher expression of polysaccharides in the entire mucosa and of MUC5AC was detected in gallbladder epithelium of 50-year-old patients, as compared to young patients with symptomatic gallstones. In the young patients, expression of polysaccharides correlated with inflammatory activity (grading), width of gallbladder wall and PLT level in peripheral blood. A significantly higher expression of polysaccharides in gallbladder epithelium was demonstrated in young patients admitted in the emergency mode to the hospital. These correlations in young patients may suggest a role of both mucus components in pathogenesis of cholelithiasis in this age group. A quantitative appraisal of mucus component expression in the two parts of gallbladder mucosa (epithelium vs. entire mucosa) using spatial visualization technique permitted to more accurately compare production of glycoproteins and of polysaccharides in patients with cholelithiasis and to demonstrate additional correlations of a potential clinical significance.
\end{abstract}

Key words: cholelithiasis, young patients, MUC5AC and polysaccharides in gallbladder mucosa, spatial visualization and quantification

\section{Introduction}

Cholelithiasis of gallbladder belongs to civilizationlinked diseases. Most frequently it is accompanied by chronic cholecystitis, which may lead to development of cancer of the organ [1]. Factors associated to a high risk of gallbladder cholelithiasis include female sex, obesity, insulin resistance, genetic conditioning and age [2-4]. Observations indicate that gallbladder cholelithiasis most frequently develops in older individuals. Never-

Correspondence: A. Kasprzak, Dept. Histology

and Embryology, University of Medical Sciences,

Święcickiego 6, 60-781 Poznań, Poland; tel.: (+4861) 8546441,

fax.: (+4861) 8546440, e-mail: akasprza@ump.edu.pl theless, in recent years an increasing incidence of symptomatic cholelithiasis is observed to affect increasingly young patients, including children $[5,6]$. Among close to three thousand patients subjected to cholecystectomy at the end of 1970ties as few as 18 were young (all of male sex), i.e. between their second and 20th year of age. Most of them manifested the idiopathic form of the disease [7]. In children and youth affected by gallstones complications and traits of choledocholithiasis used to be more frequently described [8]. Manifestation of cholelithiasis alone as well as intra- and post-operative complications in young patients used to be associated with accompanying diseases, such as inborn defects of the heart, haemolytic disease, mucoviscidosis, prolonged parenteral feeding and other diseases [9]. Risk 
factors of cholelithiasis are the same in young and in older patients. Among other, they include female sex, alimentary habits, obesity and a disturbed function of the liver [6].

Mucins produced by epithelial cells of gallbladder represent one of the key pathogenetic factors of lithogenesis. Glycoproteins of mucin are thought to be involved in preliminary stages of cholesterol gallstone formation $[10,11]$, and their overproduction intensifies development of cholesterol crystals [12,13]. Protein components of gallbladder mucins bind biliary lipids and may promote crystallization of cholesterol, contained in bile [14]. Interactions between mucins and calciumbinding proteins may result in precipitation of calcium phosphates and development of calcium-containing gallstones [15]. A role of mucins was reported in children in formation of gallstones composed of calcium carbonate [16]. Epithelium of gallbladder with gallstones was demonstrated to contain more surface mucins than epithelium of gallbladder with no gallstones [13]. Mechanisms of mucin over-production have been only partially recognised $[17,18]$. Experiments on the mouse model point to a genetic background of both mucin accumulation, cholesterol crystallization and of gallstone formation in the gallbladder [19].

Manifestation of cholelithiasis in increasingly young patients induces a justifiable interest since the aetiology remains mostly unknown, probably involving multiple factors. Better methods for an earlier diagnosis, therapy and prevention against cholelithiasis in this age group continue to be looked for.

In this study we decided to conduct analysis of tissue expression of two components of gallbladder mucus, i.e. polysaccharides and secretory mucin 5AC, as related to selected clinical data in young patients (up to 25 th year of age) with the symptomatic gallbladder cholelithiasis. Quantitative analysis of the data took advantage of the modern technique of spatial visualization, tested for its suitability in evaluation of histoand immunohistochemical reactions.

\section{Material and methods}

Patients. The studies included selected clinical data and archival tissue material (gallbladders) obtained from the patients with a symptomatic gallstones. All the patients were subjected to cholecystectomy in the T. Chałubiński Municipal Hospital in Ostrów Wielkopolski in years of 2003-2007. The principal group (group A) ( $\mathrm{n}=37$; young patients) included all patients up to 25 years of age (16 to 25 years of age, 31 women and $6 \mathrm{men}$ ) who were diagnosed and subjected to surgery in years of 2003-2007. For the reference group (group B) age criterion was accepted, amounting to approximately 50 years since in the years this was the most frequent age of cholelithiasis patients subjected to surgery at the Ward of Surgery of the mentioned above Municipal Hospital. Twenty patients were selected to the group (48 to 50 years of age, 15 women and 5 men). Duration of cholelithiasis symptoms in the analysed groups of patients most frequently ranged between 6 months and one year.
In all the patients the surgery was preceded by physical examination and USG imaging. Gallbladder cholelithiasis was diagnosed on the basis of clinical signs/symptoms and laboratory tests. In this study advantage was taken of basic laboratory results (levels of haemoglobin, WBC, PLT, bilirubin), epidemiological and/or clinical variables (age, sex, BMI). Clinical symptoms of the disease manifested upon admission to the hospital ("acute" or "chronic" ones) were also taken into account. The mode of surgery was differentiated as "urgent" or "planned". The number of gallstones removed during surgery was recorded using an arbitrary semiquantitative scale: 1- one gallstone of any size; 2- 2-20 gallstones; 3->20-100 gallstones; 4->100 gallstones. Histopathological diagnoses were established following analysis of paraffin sections (stained with hematoxylin and eosin) within routine diagnostic tests (AK) and verified in the course of this study (WB, AK). In group A 35 patients were diagnosed as affected by chronic cholecystitis and two with acute cholecystitis, in group B all the patients suffered from chronic cholecystitis.

Tissue material. The studies were conducted on serial, $5 \mu \mathrm{m}$ paraffin sections, placed on the SuperFrost/Plus microscopical slides. The archival material was fixed in a buffered $10 \%$ formalin and embedded in paraffin using the routine procedure. Studies with the use of paraffin sections included: (1) staining with hematoxylin and eosin; (2) demonstration of polysaccharides using histochemical PAS technique (periodic acid - Schiff reaction); (3) immunocytochemical reactions with the use of anti-human monoclonal antibodies specific for MUC5AC (NOVOCASTRA Labs, Ltd; Clone CLH2; Code: NCL-MUC-5AC), in dilution 1:100.

Histochemical PAS technique. Paraffin sections on microscope slides were immersed in $1 \%$ solution of periodic acid $\left(\mathrm{HJO}_{4}\right)$, washed under tap water, immersed is Schiff reagent (discoloured fuchsin) for $5 \mathrm{~min}$ and washed for $10 \mathrm{~min}$ in water. The reagent of Schiff bound aldehyde groups, yielding purple-red colour. Finally, the paraffin sections were immersed for $2 \mathrm{~min}$ in hematoxylin for counterstaining of cell nuclei, dehydrated in a row of alcohols of increasing concentration and in xylene and closed under coverslips using Canada balsam [16].

Immunocytochemical ABC technique. Detection and studies on cellular localisation of $5 \mathrm{AC}$ mucin in gallbladder mucosa took advantage of the classic ABC (strept(avidin)-biotinylated peroxidase complex) according to Hsu et al. [20], the individual stages of which were described in detail in our earlier reports [21]. Following deparaffination and rehydration the preparations were additionally boiled in $10 \mathrm{mM}$ citrate buffer in a $700 \mathrm{~W}$ microwave oven for $18 \mathrm{~min}$, washed in PBS and, then, subjected to the reaction according to the standard procedure. Every test was accompanied by a negative control in which specific antibodies were supplemented by a normal serum of a respective species in $0.05 \mathrm{M}$ Tris$\mathrm{HCl}, \mathrm{pH} \sim 7.6$ supplemented with $0.1 \%$ bovine serum albumin (BSA) and $15 \mathrm{mM}$ sodium azide.

Histological slides with polysaccharides and MUC5AC tissue expression were examined under the optical Olympus B-2 microscope coupled to a digital camera. Colour microscope images were recorded and archived using 40× objective (at least 10 fields in every microscope slide with a histochemical and/or immunocytochemical positive reaction), $2560 \times 1920$ pixels in size using LUCIA Image 5_0 computer software, documenting them in jpg format on the computer hard disc. For further processing, the images of $2560 \times 1920$ pixels in size in the LUCIA Image 5_0 software were converted to the size of $640 \times 480$ pixels. Expression of polysaccharides using the PAS technique was recorded on 680 images (characterizing 57 patients), which were subjected to a preliminary analysis of quality and image definition and/or non-specific colour reactions. Finally, the computer-assisted morphometric 

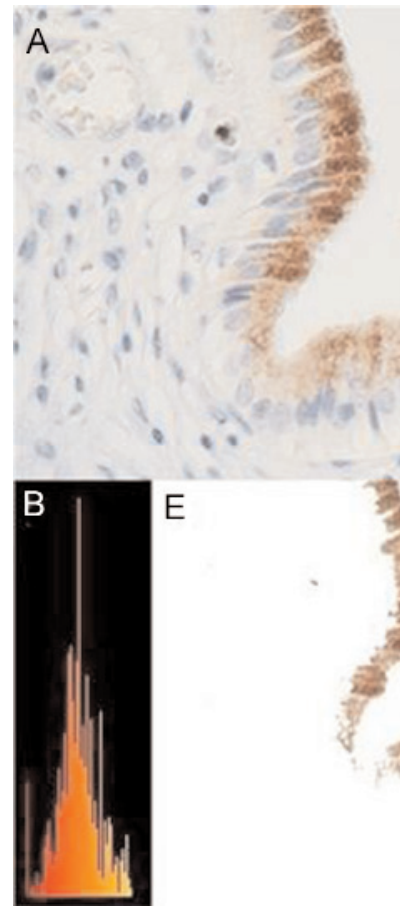

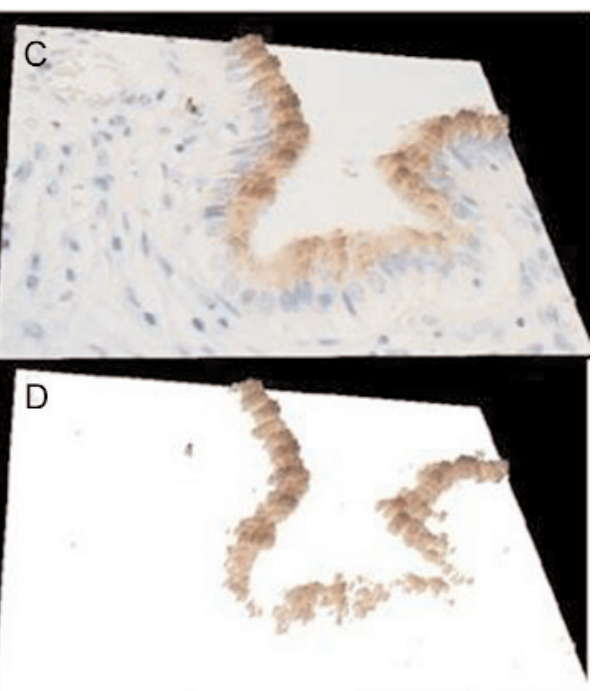

Fig. 1. Working window of A4D software. Threshold segmentation of colour immunocytochemical reaction (brown colour) on the example of mucin $5 \mathrm{AC}$ expression in gallbladder epithelium of a patient with symptomatic cholelithiasis.

A - image obtained under a light microscope coupled to a digital camera. Objective magnification: $40 \times$. ABC technique, cell nuclei counterstained with hematoxylin.

B - histogram of colours composing shades of $5 \mathrm{AC}$ mucin brown colour.

C - spatial transformation of a flat microscope image (A)

D - segmentation of 5AC mucin from a spatial image (C)

$\mathbf{E}$ - projection of the spatially transformed reaction on a plane as a final result of automatic $5 \mathrm{AC}$ mucin segmentation for measurement of its area and of its share (\%) in area of gallbladder epithelium. analysis of preparations with positive reaction for carbohydrates included 570 images. In the case of immunocytochemical detection of MUC5AC the morphometric analysis was performed on the total of 550 images.

Semi-quantitative analysis of intensity in inflammatory infiltrate in gallbladder wall (grading). Inflammatory infiltrate in a gallbladder was evaluated using a 4-grade semi-quantitative scale in which 3 points corresponded to an intense and most frequently a diffuse inflammatory infiltrate, 2 points indicated a moderately intense and also diffuse inflammatory infiltrate, 1 point indicated individual wide spread cells on inflammatory infiltrates or focally arranged cells of the infiltrate, 0 points pointed to absence of inflammatory infiltrate cells. Intensity of inflammatory infiltrate in the mucosa (epithelium and lamina propria) provided grading 1 (G1), and appropriate intensity observed in muscularis and subserous layers yielded grading 2 (G2). Total grading was also calculated, represented the sum of $\mathrm{G} 1+\mathrm{G} 2$.

Morphometric analysis. The analysis was conducted using a technique based on spatial visualization of markers in microscope images, elaborated and programmed in the A4D computer software $\mathrm{C}++$ language by Strzelczyk [22]. Application of the technique of spatial visualization to microscope images resulted in transformation of the flat image into a three-dimensional image using its brightness as the third coordinate (the spatial coordinate). The so formed spatial images contain, apart from the examined objects, also other elements, which had to be removed from the background level. For this purpose three filters were used: (1) filter of lucidity, which selects image elements the lucidity level of which does not exceed the threshold value of a lucidity histogram, typical for the analysed reaction; (2) filter of colour, in which the colour is coded as an angle of 0-360 grades, passing from the red colour, through the green and blue colours back to the red colour. This permitted to distinguish in the image colour reaction of an appropriate colour, representing a mixture of the three basic colours in image transformation: the red, green and blue colours; (3) filter of saturation, which permits to eliminate the grey background from colour images. The filters introduce no changes to the content of the orig- inal image but they mask useless elements, e.g., artefacts developed in a microscope image. Additionally, using a filter capable of smoothing median values it was possible to eliminate noises resulting from imperfect nature of CCD camera transformer, used to record microscope images.

The A4D programme allowed to sequentially calculate:

1. mean surface area of entire epithelium or entire mucosa (epithelium + lamina propria) in the recorded image (in pixels and $\mu \mathrm{m}^{2}$ ) for every patient and for the entire group of patients,

2. mean surface area of the histochemical reaction (detecting complex sugars) and immunocytochemical reaction (detecting contents of 5AC mucin) per the entire epithelial surface area or surface area of the entire mucosa (in pixels and $\mu \mathrm{m}^{2}$ ) for every patient and entire group of patients,

3. mean surface area of histochemical and/or immunocytochemical reaction for a given marker per entire epithelial area or per surface area of entire mucosa in every patient and every group of patients, calculated and expressed also in percents (\%) (Fig. 1).

Results obtained using the A4D spatial visualization software were compared with results of colour thresholding in HSI space obtained using Image J software (public domain, NHI, Bethesda, MD) applied to the same images. In this aim, the Color Thresholder plug, available at http://rsb.info.nih.gov/ij web page, was installed in the basic version of the software. The error of measuring expression of histochemical and/or immunocytochemical reaction was appraised by evaluation of the standard error of differences between measurements conducted on the same 250 microscope images but obtained using two distinct techniques (A4D and ImageJ). The standard error of mean measurement value amounted to 97 pixels or $0.97 \%$ of the mean area of reaction established by the spatial visualization technique. Thus, it might be accepted that error of the method did not exceed $1 \%$.

Results obtained in the two softwares for image analysis (LUCIA Image 50 and A4D) were exported to the format of 

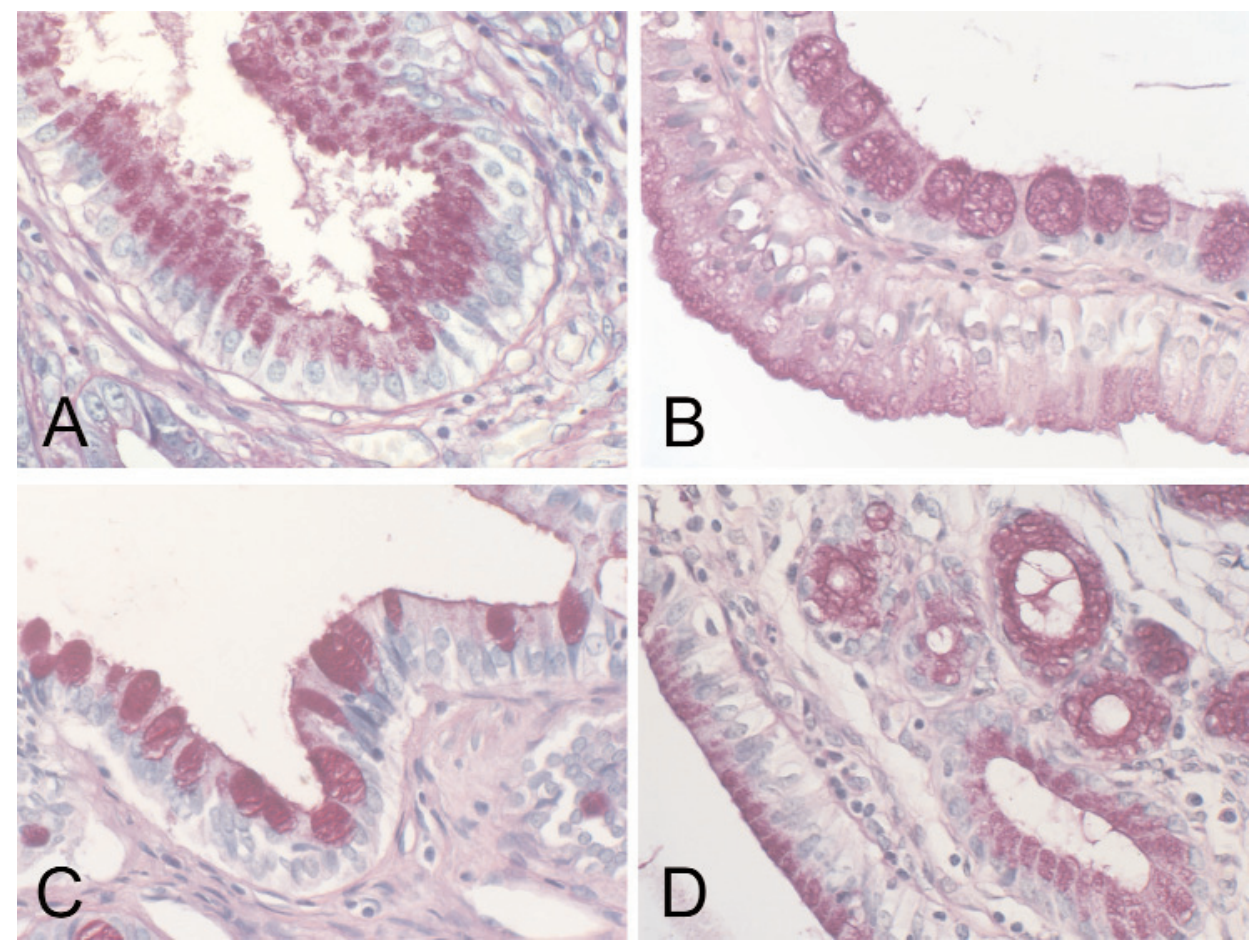

Fig. 2. A fragment of gallbladder mucosa in patients with symptomatic cholelithiasis: (A) positive reaction for polysaccharides in cytoplasm of apical portion of epithelial cells and in secretion of the cells; $(\mathrm{B}, \mathrm{C})$ localization of neutral polysaccharides in epithelial goblet cells in a young (B) and in an older patient (C); (D) presence of polysaccharides in tubulo-vesicular glands, within lamina propria. Histochemical PAS technique. Objective $40 \times$.
Microsoft Excel programme, i.e. a programme which is compatible with the Statistica PL v. 8 programme, with the aim to conduct statistical analysis. In this study results related to expression of polysaccharides and MUC5AC were presented in \% of the histochemical and immunocytochemical reactions manifested by the epithelium and the entire mucosa (epithelium + lamina propria).

Ethical issues. The study received consent of the local Bioethical Commission by Poznan University of Medical Sciences in Poznań (no. 281/08)

Statistical methods. At the first stage of the statistical analysis, compatibility of the obtained results was checked with Gauss'es distribution, using the test of Shapiro-Wilk. Employing the test of Levene, equal variances were documented for the three first parameters and, therefore, significance of differences was evaluated using the $t$ test. Subsequently, parameters of a descriptive statistics were calculated (arithmetical mean, standard deviation, median value, minimum and maximum values). Results of morphometric studies were compared between the group of A (young patients) and B (older patients, unlinked samples) using the MannWhitney's test. Spearman's rank correlation was applied to examine association between variable values. Test for two structural indices permitted to evaluate differences in detectability (\%) of selected traits between groups. Differences and relationships were accepted to be statistically significant at the level of $p \leq 0.05$. The statistical analysis took advantage of the Statistica PL v. 8 software.

\section{Results}

\section{Histochemical analysis of polysaccharides expression in gallbladder mucosa}

Detectability and cellular localization of polysaccharides. Presence of polysaccharides in epithelial cells was demonstrated as a product of a variable purple shade to an intensely violet colour. Independently of the group of patients, detectability of PAS-positive polysaccharides amounted to $100 \%$. The positive reaction was observed mainly in the apical portion of cytoplasm in most of epithelial cells (Fig. 2A). The positive reaction developed both in goblet cells and columnar cells. A particularly pronounced reaction developed in goblet cells in two patients, one in group A (Fig. 2B) and another in group B (Fig. 2C). Brush border, mucus on the surface of cells, sugar components of basement membranes (Fig. 2A) and polysaccharides of lamina propria (Fig. 2B and C) were also PAS-positive. A strong PAS-positive reaction was demonstrated in tubulo-vesicular mucus glands of lamina propria (Fig. 2D), in patients with traits of adenomyomatous hyperplasia.

\section{Morphometric evaluation of polysaccharides expression.}

Both in group A and in group B, a highly significant positive correlation was demonstrated between expression of PAS-positive polysaccharides, calculated as a $\%$ of the reaction in the epithelium alone, and expression expressed as a \% of the reaction in the entire mucosa (Table 1). A significantly higher expression of polysaccharides in the entire mucosa was demonstrated in the older patients (group B) as compared to the patients of group A (Table 2). 
Table 1. Spearman's rank correlation coefficients between expression (in $\%$ of the immunocytochemical reaction) of markers in epithelium and in the entire mucosa of gallbladder in the two groups of patients.

\begin{tabular}{|l|c|c|c|c|c|}
\hline \multicolumn{1}{|c|}{ Mucus component } & Group & $\begin{array}{c}\text { Polysaccharides } \\
\text { in epithelium }\end{array}$ & $\begin{array}{c}\text { Polysaccharides } \\
\text { in entire mucosa }\end{array}$ & $\begin{array}{c}\text { MUC5AC } \\
\text { in epithelium }\end{array}$ & $\begin{array}{c}\text { MUC5AC } \\
\text { in entire mucosa }\end{array}$ \\
\hline $\begin{array}{l}\text { polysaccharides } \\
\text { in epithelium }\end{array}$ & $\wedge$ & - & $\mathbf{0 . 7 1 7 1}$ & -0.0574 & -0.2053 \\
\cline { 2 - 6 } $\begin{array}{l}\text { polysaccharides } \\
\text { in entire mucosa }\end{array}$ & $\mathrm{B}$ & - & $\mathbf{0 . 6 3 6 8}$ & 0.1228 & -0.2870 \\
\hline \multirow{2}{*}{$\begin{array}{l}\text { MuC5 } \Lambda \mathrm{C} \\
\text { in epithelium }\end{array}$} & $\mathrm{B}$ & $\mathbf{0 . 6 3 6 8}$ & - & 0.1223 & -0.0512 \\
\hline \multirow{2}{*}{$\begin{array}{l}\text { MuC5 } \Lambda \mathrm{C} \\
\text { in entire mucosa }\end{array}$} & $\mathrm{B}$ & -0.0574 & 0.1223 & 0.1473 & -0.1270 \\
\cline { 2 - 6 } & $\mathrm{B}$ & -0.1228 & 0.1473 & - & $\mathbf{0 . 6 8 0 4}$ \\
\hline
\end{tabular}

Legend: The table contains values of Spearman's rank correlation coefficient (r); bold numbers indicate coefficients $r$ significant at $p$ (level of significance) $<0.05$;

A - group of patients below 25th year of age.; B - group of 50-year-old patients

Table 2. Expression of PAS-positive polysaccharides in patients with cholelithiasis.

\begin{tabular}{|l|c|c|c|c|c|c|}
\hline \multicolumn{1}{|c}{$\begin{array}{c}\text { Fxpression } \\
\text { of polysaccharides }\end{array}$} & Group & Mcan & SD & Min. & Max. & p \\
\hline $\begin{array}{l}\text { \% of the reaction } \\
\text { in epithelium }\end{array}$ & A & 22.30 & 11.45 & 5.38 & 58.44 & 0.1787 \\
\cline { 2 - 7 } $\begin{array}{l}\text { \% of the reaction } \\
\text { in entire mucosa }\end{array}$ & B & 26.01 & 10.90 & 10.43 & 48.05 & 41.49 \\
\cline { 2 - 7 }
\end{tabular}

Legend: bold numbers indicate statistically significant differences; $p$ - level of significance

A - group of patients below 25 th year of age.; B - group of 50-year-old patients

Table 3. Mean expression of secretory mucin MUC5AC evaluated in epithelium or entire gallbladder mucosa in the two groups of patients.

\begin{tabular}{|c|c|c|c|c|c|c|}
\hline Expression of MUC $5 \wedge \mathrm{C}$ & Group & Mean & SD & Min. & Max. & $\mathrm{p}$ \\
\hline \multirow{2}{*}{$\%$ of the reaction in epithelium } & $\Lambda$ & 11.15 & 9.35 & 0.00 & 56.17 & \multirow{2}{*}{0.0381} \\
\hline & $\mathrm{B}$ & 15.54 & 10.34 & 0.00 & 38.57 & \\
\hline \multirow{2}{*}{$\%$ of the reaction in entire mucosa } & $\mathrm{A}$ & 4.36 & 2.84 & 0.00 & 11.66 & \multirow{2}{*}{0.9044} \\
\hline & B & 4.39 & 3.08 & 0.00 & 13.12 & \\
\hline
\end{tabular}

Legend: bold numbers indicate statistically significant differences; $\mathrm{p}$ - level of significance

A - group of patients below 25 th year of age; B - group of 50-year-old patients

\section{Immunocytochemical detection of MUC5AC in gallbladder mucosa Detectability and cellular localization of MUC5AC.}

Expression of MUC5AC was detected in 36/37 (97\%) patients of group A 19/20 (95\%) patients of group B, i.e., with manifestation frequency which did not differ significantly between the groups $(p=0.7027)$. The mucin was located mainly in supranuclear parts of the cells, most frequently reaching apical portions of columnar cells in gallbladder epithelium (Figs. 3A and B). In parallel, very extensive regions of the epithelium (occasionally including a few tens of cells) which demonstrated no reaction for the protein (Fig. 3A). No expression of the protein was observed in goblet cells, present in fragments of some gallbladder epithelia in either young or older patients (Fig. 3C). In few tubulo- vesicular glands the reaction product manifested a highly variable presence or was altogether absent, or a granular reaction was observed in apical portions of individual glandular cells (Fig. 3D).

\section{Morphometric evaluation of MUC5AC expres- sion.}

Similarly to polysaccharides, a significant positive correlation was demonstrated between expression of MUC5AC, calculated as \% of the reaction in epithelium alone, and respective expression similarly calculated in the entire mucosa, both in group A and group B (Table 1). A significantly higher expression of epithelial MUC5AC was noted in gallbladders of older patients (group B) as compared to group A patients (Table 3). 

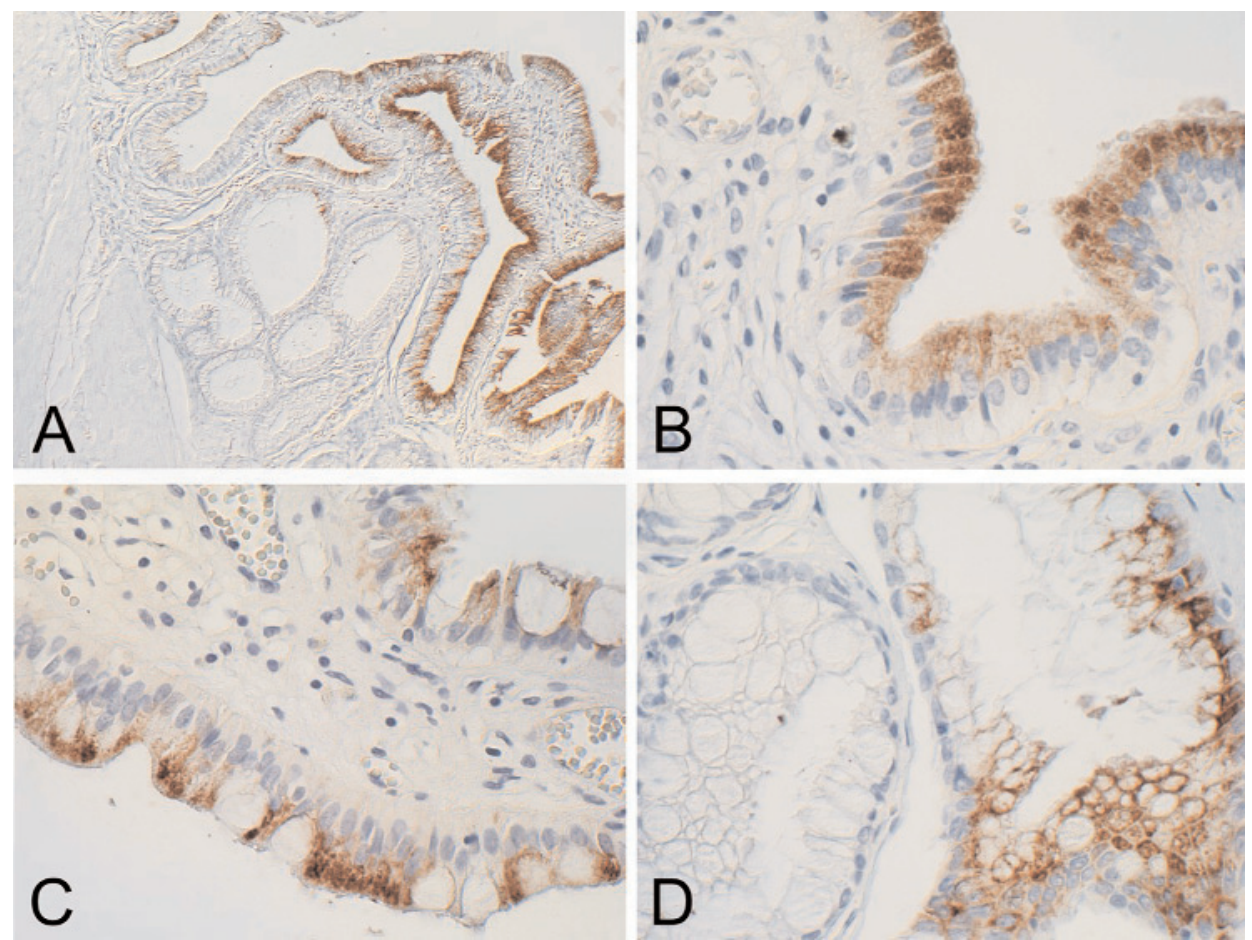

Fig. 3. Fragments of gallbladder mucosa in patients with symptomatic cholelithiasis with immunocytochemical localization of secretory 5AC mucin (MUC5AC): (A) evident positive reaction in numerous gallbladder epithelial cells in a young patient; (B) supranuclear localization of MUC5AC; (B) negative immunocytochemical reaction in goblet cells of the epithelium; (D) few epithelial cells with positive reaction in the wall of tubulo-vesicular glands, within lamina propria. ABC technique. Nomarski's optics. Objective $10 \times(\mathrm{A}) 40 \times(\mathrm{B}, \mathrm{C}, \mathrm{D})$.

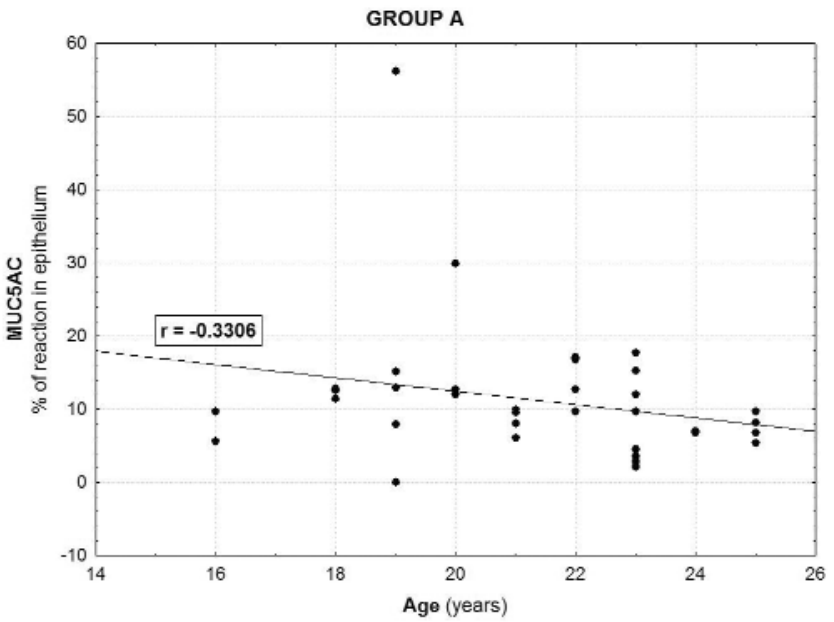

Fig. 4. Negative Spearman's correlation between epithelial expression of MUC5AC and age of patients in group A $(p<0.05)$; $r$ - Spearman's rank correlation coefficient.

\section{Correlations between expressions of polysaccharides and MUC5AC on one hand and selected clinical data on the other Marker expression and age of the patients.}

In the group of young patients (group A) a low negative correlation was detected between age of patients and expression of MUC5AC in the epithelium alone (Table 4, Fig. 4). In group B, on the other hand, the low negative correlation with age of the patients was noted for epithelial expression of PAS-positive polysaccharides (Table 5, Fig. 5).

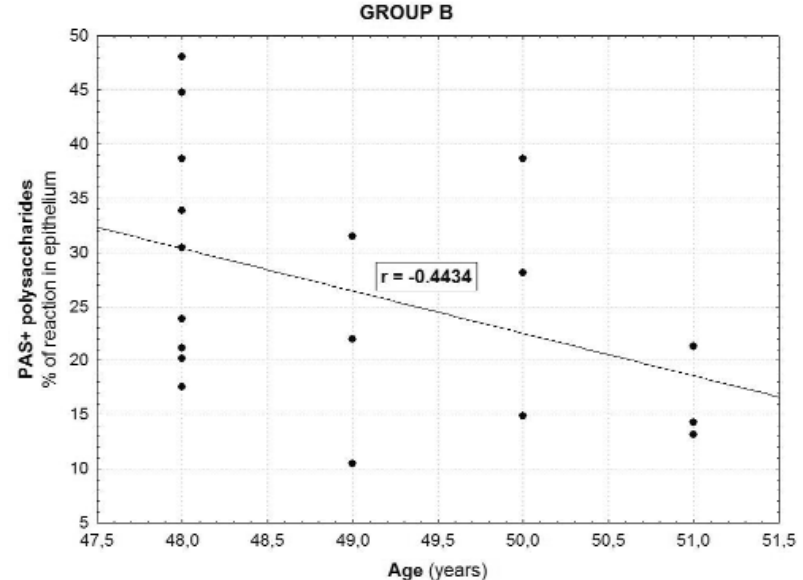

Fig. 5. Negative Spearman's correlation between epithelial expression of polysaccharides and age of patients in group $\mathrm{B}(\mathrm{p}<0.05) ; \mathrm{r}-$ Spearman's rank correlation coefficient.

BMI value. Neither in young nor in old patients could a significant correlation be discovered between BMI value and any studied marker of mucosa (Tables 4 and 5).

Number of gallstones. Neither in group A nor in group B could a significant correlation be noted between expression of polysaccharides and/or MUC5AC and a number of gallstones (Tables 4 and 5).

Width of gallbladder wall. In young patients, a positive relationship was detected between expression of polysaccharides in epithelial cells on one hand and width of gallbladder wall on the other (Table 4, Fig. 
Table 4. Spearman's rank correlation coefficient for correlation between marker expression in gallbladder mucosa and clinical data in young patients (group A).

\begin{tabular}{|c|c|c|c|c|}
\hline Trait & $\begin{array}{l}\text { Polysaccharides } \\
\text { in cpithclium }\end{array}$ & $\begin{array}{l}\text { Polysaccharides } \\
\text { in entire mucosa }\end{array}$ & $\begin{array}{c}\text { MUC5 } 5 \wedge \mathrm{C} \\
\text { in epithelium }\end{array}$ & $\begin{array}{c}\text { MUC5 } 5 \wedge \mathrm{C} \\
\text { in entire mucosa }\end{array}$ \\
\hline Age (ycars) & -0.0537 & 0.1917 & -0.3306 & -0.0367 \\
\hline $\mathrm{BM} \perp\left(\mathrm{kg} / \mathrm{m}^{2}\right)$ & -0.1597 & -0.1707 & 0.2333 & 0.1778 \\
\hline Number of gallstones & 0.0413 & 0.2160 & -0.2153 & -0.1880 \\
\hline Width of gallbladder wall (mm) & 0.3634 & 0.2729 & 0.1322 & 0.2075 \\
\hline Gil & 0.0567 & 0.0187 & 0.2128 & 0.1451 \\
\hline G2 & 0.3256 & 0.1574 & 0.1774 & 0.0239 \\
\hline $\mathrm{G} 1+\mathrm{G} 2$ & 0.2515 & 0.1340 & 0.2136 & 0.1033 \\
\hline Haemoglobin $(\mathrm{g} / \mathrm{l})$ & 0.1346 & 0.1925 & 0.1084 & 0.1636 \\
\hline $\operatorname{WBC}\left(\times 10^{\circ} / 1\right)$ & 0.1241 & 0.1165 & 0.2128 & 0.0966 \\
\hline PI,T $\left(\times 10^{\circ} / 1\right)$ & -0.3605 & -0.2263 & 0.1724 & 0.2724 \\
\hline Bilirubin (mg/dl) & 0.3659 & -0.0623 & 0.1606 & -0.1545 \\
\hline
\end{tabular}

Legend: The table presents values of the coefficient of Spearman's rank correlation (r); bold numbers indicate values of $r$ significant at $p<0.05$; BMI- body mass index; G - grading; WBC- white blood cells; PLT - platelets

Table 5. Spearman's rank correlation coefficients for expression of markers in gallbladder mucosa and clinical variables in older patients (group B).

\begin{tabular}{|c|c|c|c|c|}
\hline Trait & $\begin{array}{l}\text { Polysaccharides } \\
\text { in cpithclium }\end{array}$ & $\begin{array}{l}\text { Polysaccharides } \\
\text { in cntire mucosa }\end{array}$ & $\begin{array}{c}\text { MUC5 } 5 \mathrm{C} \\
\text { in epithelium }\end{array}$ & $\begin{array}{c}\text { MUC5 } 5 \wedge \mathrm{C} \\
\text { in entire mucosa }\end{array}$ \\
\hline Age (years) & -0.4434 & -0.2723 & 0.1433 & 0.2670 \\
\hline BMI $\left(\mathrm{kg} / \mathrm{m}^{2}\right)$ & 0.2132 & 0.1220 & 0.2648 & 0.1124 \\
\hline Number of gallstones & 0.0066 & 0.2657 & -0.0768 & 0.0135 \\
\hline Width of gallbladder wall (mm) & 0.2624 & -0.0426 & 0.2990 & 0.1747 \\
\hline $\mathrm{Gil}$ & 0.4638 & 0.2918 & 0.2232 & -0.0373 \\
\hline $\mathrm{G} 2$ & 0.5331 & 0.1185 & 0.3976 & -0.0787 \\
\hline $\mathrm{Gil}+\mathrm{G} 2$ & 0.5036 & 0.2089 & 0.3128 & -0.0657 \\
\hline Haemoglobin $(\mathrm{g} / \mathrm{l})$ & 0.1970 & 0.0317 & 0.0739 & 0.1802 \\
\hline WBC $\left(\times 10^{9} / 1\right)$ & 0.4223 & 0.5154 & 0.0730 & -0.1779 \\
\hline PL.T $\left(\mathrm{x} 10^{9} / 1\right)$ & 0.3842 & 0.4754 & -0.1549 & -0.2027 \\
\hline Bilirubin (mg/dl) & 0.1495 & -0.0309 & -0.0124 & 0.1892 \\
\hline
\end{tabular}

Legend: The table presents values of Spearman's rank correlation coefficient $(r)$; bold numbers indicate $r$ values significant at $p<0.05$; BMI- body mass index; G - grading; WBC- white blood cells; PLT - platelets

6A). No such a relationship could be detected for expression of MUC5AC in the group of patients. In the group of 50-year-old patients no correlation was noted between width of gallbladder an expression of any studied mucosal marker (Table 5).

Grading. In patients up to 25 years of age, increasing activity of inflammatory lesions in deeper layers of gallbladder wall $(\mathrm{G} 2)$ was found to be associated with the growing expression of polysaccharides in gallbladder epithelium (Table 4; Fig. 6B). In the older patients a positive correlation was noted between epithelial expression of polysaccharides on one hand, G1, G2 values and total grading $(\mathrm{G} 1+\mathrm{G} 2)$ on the other (Table 5; Fig. 7). In the studied groups of patients no significant correlations could be detected between grading
$(\mathrm{G} 1, \mathrm{G} 2, \mathrm{G} 1+\mathrm{G} 2)$ and expression of MUC5AC.

Number of WBC. None of the studied tissue markers was found to manifest a significant correlation with WBC in the group of younger patients (Table 4). In 50year-old patients level of WBC demonstrated a positive correlation with expression of polysaccharides in the entire mucosa (Table 5).

Blood platelet level. In younger patients clearly higher number of blood platelets was accompanied by a lower expression of polysaccharides in gallbladder epithelium (Table 4; Fig. 6C), while in the older patients higher number of blood platelets manifested a positive correlation with expression of carbohydrates in the entire mucosa (Table 4). Expression of MUC5AC manifested no significant correlations with PLT number in 

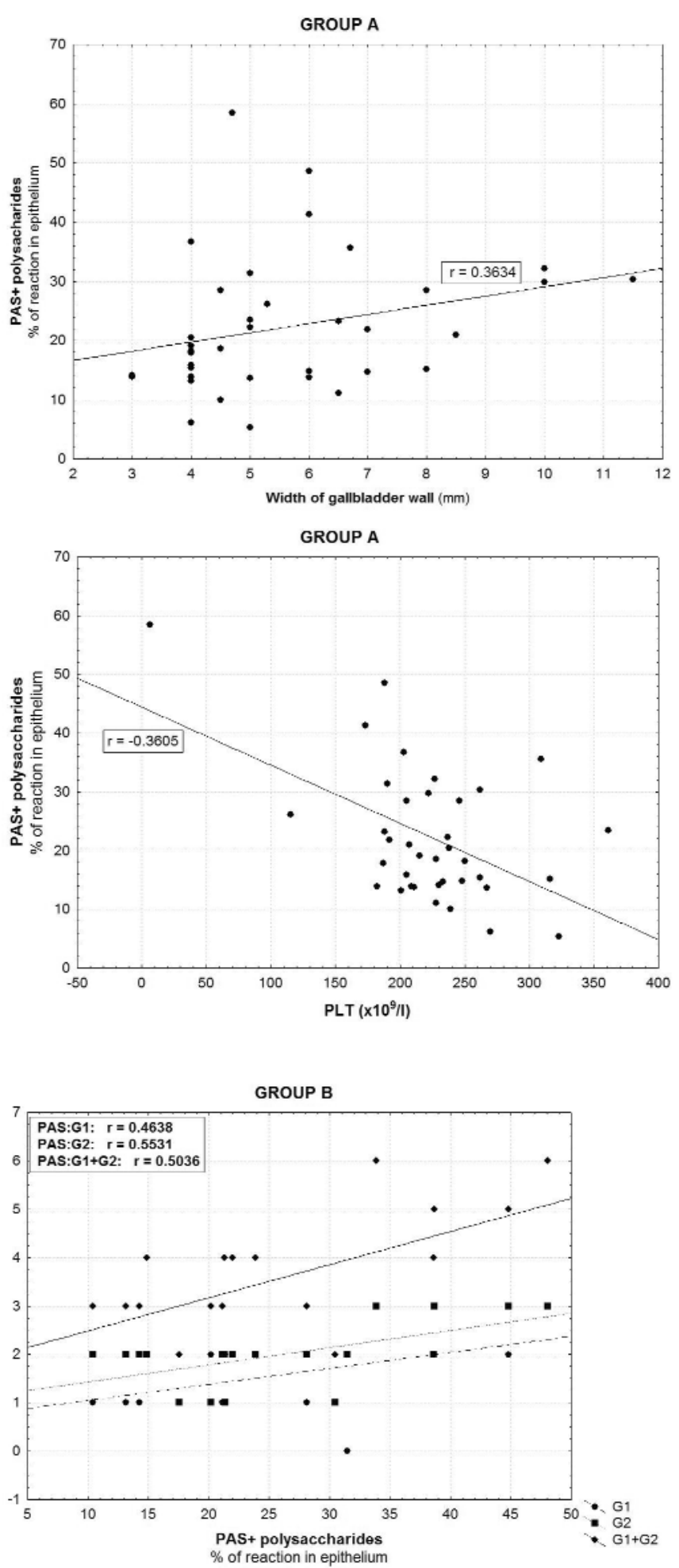

Fig. 7. Positive Spearman's correlation between epithelial expression of polysaccharides in patients of group B and activity of inflammation in all layers of gallbladder wall $(\mathrm{G} 1, \mathrm{G} 2)$ and total grading $(\mathrm{G} 1+\mathrm{G} 2)$; $r$ - Spearman's rank correlation index.

any studied group of patients (Tables 4 and 5).

Expression of markers and acute or chronic clinical symptoms. In young patients no statistically significant differences were detected either in expression of poly-

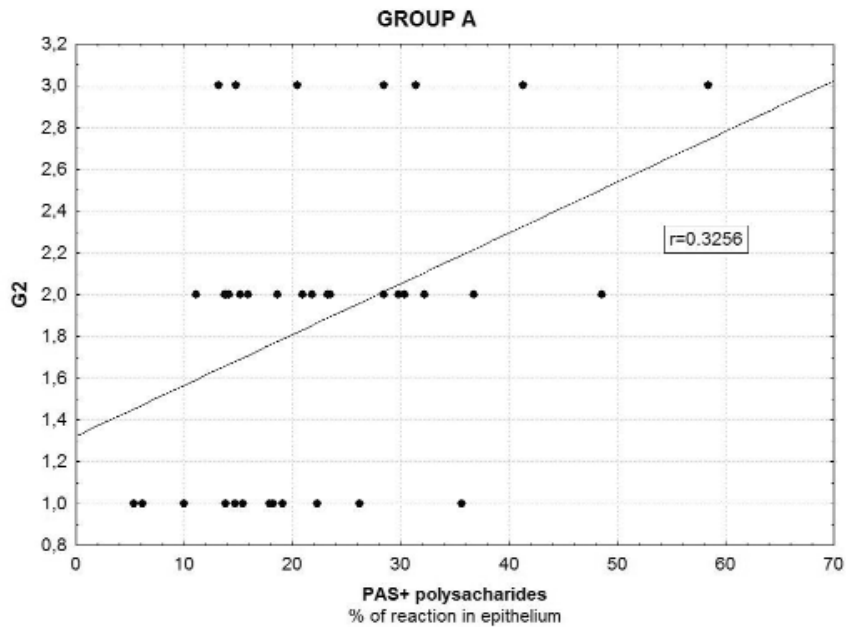

Fig. 6. Positive Spearman's correlation between epithelial expression of PAS-positive polysaccharides in patients of group A and: (A) width of gallbladder wall; (B) activity of inflammation in deeper layers of gallbladder wall (G2); (C) negative correlation between epithelial expression of PAS-positive polysaccharides and number of blood platelets; $(\mathrm{p}<0.05$ in all cases $) ; \mathrm{r}$ - Spearman's rank correlation index.

saccharides or MUC5AC as related to an acute or chronic course of clinical symptoms (Table 6). In the group of older patients a significantly higher expression of MUC5AC in epithelium alone was noted in patients with acute, as compared to those with chronic, clinical symptoms (Table 7).

As related to the emergency versus planned mode of admission, the group of young patients admitted in the emergency mode demonstrated a significantly higher expression of polysaccharides as compared to patients admitted in the planned mode (Table 6).

\section{Reciprocal correlation between expressions of polysaccharides and of MUC5AC}

In young patients (group A) higher expression of polysaccharides, demonstrated using the PAS histochemical technique, coexisted with the higher expression of secretory mucin (MUC5AC), detected by the immunocytochemical $\mathrm{ABC}$ technique, but this could be documented not for the expression values expressed in \% of the reaction (Table 1), but for the expression appraised morphometrically in pixels and per $\mu \mathrm{m}^{2}$ of gallbladder 
Table 6. Values of mean marker expression in young patients (group A) as related to clinical symptoms and mode of admission to hospital.

\begin{tabular}{|c|c|c|c|c|c|c|c|}
\hline Marker & $\begin{array}{l}\text { Symptoms/mode } \\
\text { of admission } \\
\text { to the hospital }\end{array}$ & $\mathrm{n}$ & Mcan & $\mathrm{SD}$ & Min. & Max. & $\mathrm{p}$ \\
\hline \multirow{4}{*}{$\begin{array}{l}\text { Polysaccharides } \\
\text { in epithelium }\end{array}$} & acute & 12 & 27.85 & 13.20 & 58.44 & 14.32 & \multirow{2}{*}{0.1019} \\
\hline & chronic & 25 & 19.63 & 5.38 & 41.30 & 8.94 & \\
\hline & emergency & 15 & 29.35 & 13.20 & 58.44 & 7.24 & \multirow{2}{*}{0.0033} \\
\hline & planned & 22 & 17.49 & 5.38 & 36.74 & 12.99 & \\
\hline \multirow{4}{*}{$\begin{array}{l}\text { Polysaccharides } \\
\text { in entire mucosa }\end{array}$} & acute & 12 & 16.40 & 11.78 & 3.80 & 41.49 & \multirow{2}{*}{0.9109} \\
\hline & chronic & 25 & 14.16 & 7.66 & 2.38 & 34.43 & \\
\hline & emergency & 15 & 17.92 & 11.34 & 3.80 & 41.49 & \multirow{2}{*}{0.2248} \\
\hline & planned & 22 & 12.82 & 6.71 & 2.38 & 29.41 & \\
\hline \multirow{4}{*}{$\begin{array}{l}\text { MUC5AC } \\
\text { in epithelium }\end{array}$} & acute & 12 & 15.77 & 14.75 & 0.00 & 56.17 & \multirow{2}{*}{0.0825} \\
\hline & chronic & 25 & 8.93 & 3.96 & 2.13 & 16.80 & \\
\hline & emergency & 15 & 13.78 & 13.89 & 0.00 & 56.17 & $0-5107$ \\
\hline & planned & 22 & 9.35 & 3.62 & 2.81 & 16.80 & 8.010 \\
\hline \multirow{4}{*}{$\begin{array}{l}\text { MUC5 } 5 \wedge \mathrm{C} \\
\text { in entire mucosa }\end{array}$} & acute & 12 & 5.09 & 3.62 & 0.00 & 11.66 & \multirow{2}{*}{0.4908} \\
\hline & chronic & 25 & 4.00 & 2.39 & 0.84 & 7.82 & \\
\hline & emergency & 15 & 4.34 & 3.54 & 0.00 & 11.66 & \multirow{2}{*}{0.5716} \\
\hline & planned & 22 & 4.36 & 2.35 & 0.84 & 7.82 & \\
\hline
\end{tabular}

Legend: bold number indicates a statistically significant difference; $p$ - level of significance

Table 7. Mean values of marker expression in older patients (group B) as related to clinical symptoms and mode of admission to hospital.

\begin{tabular}{|c|c|c|c|c|c|c|c|}
\hline Marker & $\begin{array}{c}\text { Symptoms/mode } \\
\text { of admission } \\
\text { to the hospital }\end{array}$ & $\mathrm{n}$ & Mean & SD & Min. & Max. & $\mathrm{p}$ \\
\hline \multirow{4}{*}{$\begin{array}{l}\text { Polysaccharides } \\
\text { in epithelium }\end{array}$} & acute & 9 & 28.30 & 14.27 & 48.05 & 13.19 & \multirow{2}{*}{0.5490} \\
\hline & chronic & 10 & 23.94 & 10.43 & 38.64 & 8.55 & \\
\hline & emergency & 9 & 26.02 & 10.43 & 48.05 & 13.23 & \multirow{2}{*}{0.9682} \\
\hline & planned & 10 & 25.99 & 13.14 & 38.68 & 9.07 & \\
\hline \multirow{4}{*}{$\begin{array}{l}\text { Polysaccharides } \\
\text { in entire mucosa }\end{array}$} & acute & 9 & 28.27 & 12.38 & 8.87 & 44.11 & \multirow{2}{*}{0.0788} \\
\hline & chronic & 10 & 17.89 & 4.29 & 8.25 & 21.49 & \\
\hline & emergency & 9 & 25.36 & 11.36 & 8.87 & 42.94 & \multirow{2}{*}{0.3562} \\
\hline & planned & 10 & 20.51 & 9.18 & 8.25 & 44.11 & \\
\hline \multirow{4}{*}{$\begin{array}{l}\text { MUC5AC } \\
\text { in epithelium }\end{array}$} & acute & 10 & 21.89 & 9.12 & 10.71 & 38.57 & \multirow{2}{*}{0.0038} \\
\hline & chronic & 10 & 9.19 & 7.26 & 0.00 & 21.74 & \\
\hline & emergency & 10 & 18.41 & 11.75 & 0.32 & 38.57 & 02030 \\
\hline & planned & 10 & 12.68 & 8.32 & 0.00 & 24.74 & טיס \\
\hline \multirow{4}{*}{$\begin{array}{l}\text { MUC5AC } \\
\text { in entire mucosa }\end{array}$} & acute & 9 & 5.59 & 3.04 & 2.79 & 13.12 & \multirow{2}{*}{0.1127} \\
\hline & chronic & 10 & 3.32 & 2.82 & 0.00 & 8.81 & \\
\hline & emergency & 9 & 4.22 & 1.87 & 0.00 & 6.62 & \multirow{2}{*}{0.7197} \\
\hline & planned & 10 & 4.55 & 3.97 & 0.00 & 13.12 & \\
\hline
\end{tabular}

Legend: bold number indicates a statistically significant difference; $p$ - level of significance 


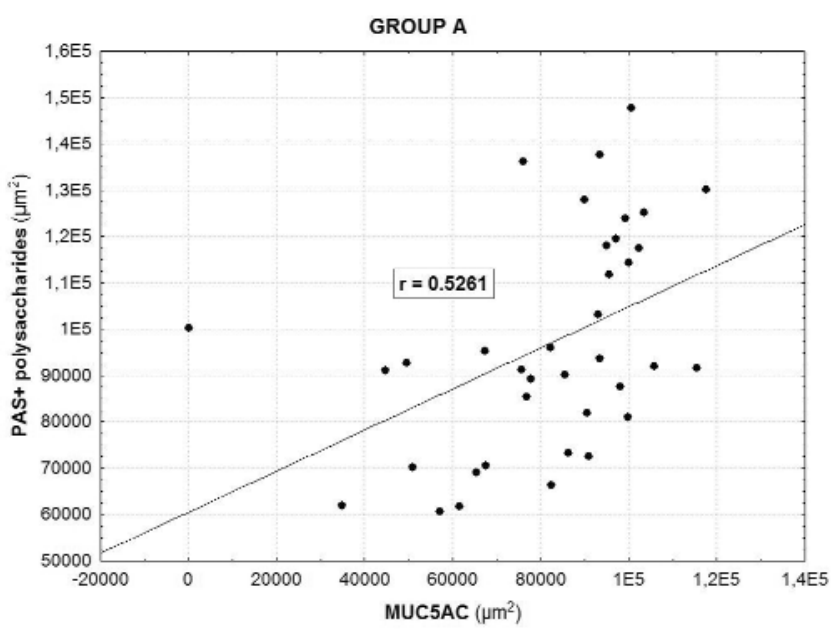

Fig. 8. Positive Spearman's rank correlation between expression of complex PAS-positive polysaccharides and expression of MUC5AC in gallbladder epithelium in patients of group A; $r$ Spearman's rank correlation index.

epithelium (Fig. 8). No such a correlation could be documented between the studied mucus components in the group of older patients (group B) (Table 1).

\section{Discussion}

PAS technique used to be applied for evaluation of production of mucus by gallbladder epithelial cells in various species of animals and in humans in normal and pathological conditions $[23,24]$. Few of these reports pertained to gallstone cholecystitis in humans $[16,25]$. In normal conditions gallbladder epithelium produced the so called sulphomucin while in cholecystitis the mucus was observed to undergo carboxylation and the mucus was so called neutral (or a mixture of the two types of mucus was noted) [23]. An increased content of glycogen was noted in more pronounced inflammatory lesions in gallbladder, as compared to less pronounced lesions, while effective therapy resulted in return of the normal mucus [25].

In this study, application of the modern technique of spatial visualization to evaluation of polysaccharides and secretory MUC5AC mucin in gallbladder mucosa in the two groups of patients provided a reliable quantitative analysis of expression of the mucus components using histochemical and immunocytochemical approach. Higher expression of both mucus components (neutral polysaccharides and MUC5AC) has been demonstrated in older patients as compared to young patients. Moreover, expression of polysaccharides has been shown to increase in parallel to increasing grading in gallbladder wall, which could have been observed in particular in the group of 50-year-old patients (correlations with both G1 and G2 values). In young patients expression of polysaccharides has been demonstrated to manifest positive correlation with width of gallbladder wall. The almost twice higher expression of polysaccharides in the epithelium alone has been documented in young patients admitted in the emergency mode as compared patients submitted to planned operations. Other authors documented a relationship between an increased (as compared to the control) production of PAS-positive mucus in epithelial gallbladder cells in children with gallstones consisting of calcium carbonate, suggesting pathogenetic relationship of this type of gallstones in the youngest patients [16]. Results of this study cannot be compared to this only report since we have had no data as to the type of gallbladder deposits and we have studied a group of slightly older patients (mean age of around 21 years as compared to around 8 years in the quoted report). Also we have had no tissue material from fully healthy patients of respective age (ethical reasons). Nevertheless, demonstration of positive correlations between augmented expression of polysaccharides and an increased severity of inflammatory lesions in gallbladder, acute clinical course and/or emergency mode of the surgery may, at least partially, confirm involvement of PAS-positive neutral polysaccharides in pathogenesis of cholelithiasis and clinical course of the disease.

The secretory MUC5AC mucin (as a component of mucus) protects gallbladder epithelial cells from exposure to biliary salts. It is produced mainly by epithelial cells and secreted to an extracellular space [26]. Protein components of mucin are assumed to bind biliary lipids and to promote crystallization of biliary cholesterol [14]. In turn, interactions of mucins with calcium-binding proteins is supposed to promote precipitation of calcium phosphates and formation of calcium gallstones [15]. Scientific reports (few until now) point to alterations in expression of mucins in various diseases of gallbladder $[13,27,28]$. In $86-100 \%$ normal gallbladders a dominating expression of MUC3, MUC5AC, MUC5B and MUC6 mucins was demonstrated. In epithelial cells MUC3, MUC5AC and MUC5B prevailed while MUC5B and MUC6 dominated in deeper layers of gallbladder mucosa [28]. The group of Sasaki demonstrated over-expression of MUC2, MUC3, MUC5AC, MUC5B and MUC6, but in cholelithiasis of intrahepatic biliary ducts, suggesting a causal relationship between, in particular, MUC2 and MUC5AC and development of biliary stones [27].

In this study expression of MUC5AC has been documented in almost all (with a single exception in each of group A and B) gallbladders of the patients, independently of the group. The glycoprotein has been located mainly in apical portions of gallbladder epithelial cells, which corroborates observations of other authors $[16,18]$. Depending on epithelial region and individual traits of the patient a variable intensity of 
the immunocytochemical reaction has been observed. The immunocytochemical reaction has been negative in goblet cells present in some fragments of epithelia in both groups of the patients. As a rule no MUC5AC expression has been noted (or it has been very low) in hyperplastic cells of clear cell glands in lamina propria. This is consistent with observations of other authors, related to chemical composition of mucus in columnar cells of gallbladder epithelium in cholecystitis (containing mainly neutral mucus) as compared to the secretion of goblet cells or gland cells (producing mainly acidic mucus) $[16,23,24]$.

Mean value of MUC5AC expression has not been high (on the average below $5 \%$ of the reaction in the entire mucosa and around $15 \%$ of the reaction in epithelium) but, as already mentioned above, the higher amounts of the epithelial mucin has been detected in 50-year-old patients as compared to young patients. In the older patients who upon admission demonstrated acute clinical symptoms epithelial expression of MUC5AC has been twofold higher than in the patients with chronic symptoms. It can be recalled that similar clinical observations have pertained to expression of polysaccharides in the group of patients. It should be mentioned that the MUC5AC molecule consists in $80 \%$ of sugar residues $[26,29]$. Mucins (including polymeric, secreted mucin MUC5AC) are complex O-linked glycoproteins characterized by the presence of at least one large region of the polypeptide rich in serine and threonine residues, termed the mucin domain. These hydroxylated amino acids are the sites of covalent attachment, via the linkage sugar $\mathrm{N}$-acetylgalactosamine of a diverse array of O-glycans, many of which are sialylated or sulphated. As a consequence mucins are polyanionic, and a high percentage of their mass is cardohydrate [29].

In this study we have been able to demonstrate parallelism of a higher epithelial expression of MUC5AC with a more pronounced expression of polysaccharides but this has been so just in the young cholelithiasis patients. The result might suggest an interrelationship between productions of the two distinct mucus components (sugars, proteins) by gallbladder epithelial cells in cholecystitis accompanied by gallstones. Nevertheless, in none of the studied groups the studies could have confirmed a correlation between expression of MUC5AC and grading of inflammation in the wall of gallbladder. As mentioned above, quantitative comparison of epithelial expressions of MUC5AC and polysaccharides has demonstrated a significantly higher expression of both mucus components in the older as compared to the young patients. This might point to a compensatory production of the two mucus components forming a protective layer of the mucosa but, on the other hand, the higher amount of mucus might have promoted formation of gallstones, particularly in the older patients.

Due to the relatively low patient's age variability both in the group of young and that of older patients it remains difficult to interpret relatively controversial results pointing to a negative correlation between patient's age and MUC5AC (in young patients) or PAS-positive polysaccharides (50-year-old patients). The result would require confirmation in the patients' population with a higher age range.

As related to the other data, it has been confirmed that MUC5AC belongs to principal mucins produced by gallbladder epithelium. Employing imunocytochemistry to localize several mucins in patients with cholelithiasis, several authors detected the highest amounts of MUC5AC and MUC5B [28,30]. However, we have not been able to confirm the observations that a higher expression of MUC5AC is accompanied by more pronounced inflammatory lesions in gallbladder [30]. Other authors demonstrated a decreased expression of MUC5AC in epithelium in cases of acute cholecystitis as compared to the normal epithelium [28]. We could not have confirmed the observation since morphological traits of acute cholecystitis have been detected just in two examined patients.

\section{Summary and conclusions}

The results of our studies point to high usefulness of spatial visualization in evaluation of both immunocytochemcial and histochemical reactions in tissue material. A quantitative appraisal of mucus component expression in the two parts of gallbladder mucosa (epithelium vs. entire mucosa) permitted to more accurately compare production of glycoproteins and of polysaccharides in patients with symptomatic cholelithiasis and to demonstrate additional correlations of a potential clinical significance. The morphometric studies documented a positive correlation between tissue expression of polysaccharides, demonstrated using the PAS technique, with tissue expression of MUC5AC in young patients, which points to involvement of both mucus components in pathogenesis of a symptomatic cholelithiasis in patients below 25 years of age.

Acknowledgments: Source of support: a grant from the Rector of Poznan Medical University, Poznań, Poland (no. 501-0202229373-05360-50502).

\section{References}

[ 1] Kumar S, Kumar S, Kumar S. Infection as a risk factor for gallbladder cancer. J Surg Oncol. 2006;93:633-639.

[2] Schirmer BD, Winters KL, Edlich RF. Cholelithiasis and cholecystitis. J Long Term Eff Med Implants. 2005;15:329-38.

[3] Hernandez-Nazara A, Curier-Lopez F, Martinez-Lopez E, Hernandez-Nazara Z, Panduro A. Genetic predisposition of cholesterol gallstone disease. Ann Hepatol. 2006;5:140-149. 
[ 4] Mathur A, Al-Azzawi HH, Lu D et al. Steatocholecystitis: the influence of obesity and dietary carbohydrates. J Surg Res. 2008; 147:290-297.

[ 5] Bailey PV, Connors RH, Tracy TF Jr, Sotelo-Avila C, Lewis JE, Weber TR. Changing spectrum of cholelithiasis and cholecystitis in infants and children. Am $J$ Surg. 1989;158:585-588.

[6] Lugo-Vicente HL. Trends in management of gallbladder disorders in children. Pediatr Surg Int. 1997;12:348-352.

[ 7] Haff RC, Andrassy RJ, LeGrand DR, Ratner IA. Gallbladder disease in the young male. Am J Surg. 1976;131:232-234.

[ 8] Waldhausen JH, Benjamin DR. Cholecystectomy is becoming an increasingly common operation in children. Am J Surg. 1999; 177:364-367.

[ 9] Miltenburg DM, Schaffer R, Breslin T, Brandt ML. Changing indications for pediatric cholecystectomy. Pediatrics. 2000;105:1250-1253.

[10] Klomp LWJ, De Lely AJ, Strous GJ. Biosynthesis of a human gall-bladder mucin. Biochem J. 1994;304:737-744.

[11] Madrid JF, Hernandez F, Ballesta J. Characterization of glycoproteins in the epithelial cells of human and other mammalian gallbladder. A review. Microsc Res Tech. 1997;15:616630.

[12] Corradini SG, Liguori F. Recent studies on the pathogenesis of cholelithiasis: the role of the gallbladder epithelium. Recenti Prog Med. 2001;92:471-476.

[13] Lee KT, Liu TS. Mucin gene expression in gallbladder epithelium. J Formos Med. Assoc 2002;101:762-768.

[14] Smith BF. Human gallbladder mucin binds biliary lipids and promotes cholesterol crystal nucleation in model bile. J Lipid Res. 1987;28:1088-1097.

[15] Afdhal NH, Niu N, Gantz D, Small DM, Smith BF. Bovine gallbladder mucin accelerates cholesterol monohydrate crystal growth in model bile. Gastroenterology. 1993;104:15151523.

[16] Sayers C, Wyatt J, Soloway RD, Taylor DR, Stringer MD. Gallbladder mucin production and calcium carbonate gallstones in children. Pediatr Surg Int. 2007;23:219-223.

[17] Dray-Charier N, Paul A, Combettes L, et al. Regulation of mucin secretion in human gallbladder epithelial cells: predominant role of calcium and protein kinase C. Gastroenterology. 1997;112:987-990.

[18] Finzi L, Barbu V, Burgel P-R, et al. MUC5AC a gel-forming mucin accumulating in gallstone disease, is overproduced via an Epidermal Growth Factor Receptor pathway in the human gallbladder. Am J Pathol. 2006;169:2031-2041.
[19] Lammert F, Wang DQ-H, Wittenburg H, et al. Lith genes control mucin accumulation, cholesterol crystalization, and gallstone formation in $\mathrm{A} / \mathrm{J}$ and $\mathrm{AKR} / \mathrm{J}$ inbred mice. Hepatology. 2002;36:1145-1154.

[20] Hsu SM, Raine L, Fanger H. Use of avidin-biotin-peroxidase complex $(\mathrm{ABC})$ in immunoperoxidase techniques. $J$ Histochem Cytochem. 1981;29:577-580.

[21] Kasprzak A, Adamek A, Przybyszewska W, et al. p53 immunocytochemistry and TP53 gene mutations in patients with chronic hepatitis $\mathrm{C}$ virus (HCV) infection. Folia Histochem Cytobiol. 2009;47:5-12.

[22] Kaczmarek E, Strzelczyk R. From two to three-dimensional visualisation of structures in light and confocal microscopy applications for biomedical studies. In: Mendez-Vilas A, Labajos-Broncano L (eds.): Current issues on multidisciplinary microscopy research and education. FORMATEX microscopy book series no. II Formatex Research Centre, Badajoz, pp 289-295.

[23] Esterly JR, Spicer SS. Mucin histochemistry of human gallbladder: changes in adenocarcinoma, cystic fibrosis, and cholecystitis. J Natl Cancer Inst. 1968;40:1-12.

[24] Yamada K, Hoshino M. Morphochemical analysis of epithelial mucosubstances in the gall bladder of the fowl (Gallus domesticus). Histochemie. 1972;29:120-128.

[25] Kiumurieva-Lazarova V. Cytochemical study of glycogen and peroxidase in acute and chronic exacerbated cholecystitis uncomplicated and complicated by cholelithiasis. Vnutr Boles. 1984;23:112-115.

[26] Gendler SJ, Spicer AP. Epithelial mucin genes. Annu Rev Physiol. 1995;57:607-634.

[27] Sasaki M, Nakanuma Y, Kim YS. Expression of apomucins in the intrahepatic biliary tree in hepatolithiasis differ from that in normal liver and extrahepatic biliary obstruction. Hepatology. 1998;27:46-53.

[28] Ho SB, Shekels LL, Toribara NW, et al. Altered mucin core peptide expression in acute and chronic cholecystitis. Dig Dis Sci. 2000;45:1061-1071.

[29] Thornton DJ, Rousseau K, McGuckin A. Structure and function of the polymeric mucins in airways mucus. Annu Rev Physiol. 2008;70:459-486.

[30] Vilkin A, Nudelman I, Morgenstern S, et al. Gallbladder inflammation is associated with increase in mucin expression and pigmented stone formation. Dig Dis Sci. 2007;52:16131620 . 